\title{
Estimating the Costs of Opioid Abuse and Dependence from an Employer Perspective: a Retrospective Analysis Using Administrative Claims Data
}

\author{
J. Bradford Rice • Noam Y. Kirson - Amie Shei • \\ Alice Kate G. Cummings $\cdot$ Katharine Bodnar • \\ Howard G. Birnbaum · Rami Ben-Joseph
}

Published online: 12 June 2014

(C) Springer International Publishing Switzerland 2014

\begin{abstract}
Background Opioid abuse and dependence is problematic across many age groups, including the working-age population and their dependents. Little is known, however, about the economic costs of opioid abuse/dependence imposed on employers, who pay for a substantial portion of healthcare costs through their contributions to employersponsored health insurance and are also affected by indirect costs such as those due to disability and workplace absenteeism.

Objective To provide a comprehensive, current estimate of the economic burden of prescription opioid abuse/ dependence to employers.

Methods Administrative claims from beneficiaries covered by large self-insured companies throughout the USA were used to identify patients, including employees and dependents, who were diagnosed with opioid abuse and/or dependence ('abusers') between 2006 and 2012. Healthcare and work-loss costs for abusers were assessed over a 12-month period and compared with those for patients not diagnosed with abuse ('comparison patients'), using propensity score matching.

Results 7,658 matched pairs of abusers and comparison patients were analysed. Relative to comparison patients, abusers had significantly higher annual healthcare resource utilization, leading to US\$10,627 in per-patient
\end{abstract}

J. B. Rice $(\bowtie) \cdot$ N. Y. Kirson · A. Shei ·

A. K. G. Cummings - K. Bodnar - H. G. Birnbaum

Analysis Group, Inc., 111 Huntington Avenue, 10th Floor,

Boston, MA 02199, USA

e-mail: brice@analysisgroup.com

R. Ben-Joseph

Purdue Pharma L.P., 1 Stamford Forum, Stamford,

CT 06901, USA incremental annual healthcare costs. Additionally, abusers had US $\$ 1,244$ in excess annual work-loss costs. Together, this implies an employer burden for diagnosed abuse of US $\$ 1.71$ per member per month.

Conclusion Opioid abuse/dependence impose a substantial economic burden on employers.

\section{Key Points for Decision Makers}

Diagnosed opioid abuse/dependence results in substantial excess healthcare and work-loss costs

The prevalence of diagnosed opioid abuse/ dependence has increased from 2006 to 2012

\section{Introduction}

\subsection{Background}

Pain is a highly prevalent condition. The Institute of Medicine estimates that over 100 million US adults suffer from chronic pain due to medical conditions, such as arthritis, back pain, migraine headaches and postoperative pain [1]. Many of these conditions affect mental and physical functioning, often severely restricting the ability to perform daily routines [2]. To treat pain, physicians have increasingly turned to prescription opioid analgesics, which are among the most effective drugs for pain management [3]. As a result, opioids have become some of the most frequently prescribed medications in the USA, with over 235 million prescriptions dispensed in 2011, 
corresponding to US\$8.3 billion in sales [4]. These medications, however, also carry the risk of potential abuse, unintended misuse and dependence. According to the National Survey on Drug Use and Health (NSDUH), the number of Americans who abused or were dependent upon pain relievers increased from 1.6 to 1.8 million between 2006 and $2011[5,6]$. The NSDUH is a primary source of information on the use of illicit drugs, alcohol and tobacco in the civilian, non-institutionalized population of the USA aged 12 years or older, and questions about illicit drug and alcohol dependence and abuse are based on criteria in the Diagnostic and Statistical Manual of Mental Disorders, 4th edition (DSM-IV). In order to be consistent with the NSDUH, we combine opioid abuse and dependence in this article. For simplicity, throughout this manuscript, we use the term 'abuse' to describe the combination of abuse and dependence.

Opioid abuse is problematic across many age groups, including the working-age population and their dependents [7]. For those individuals, employers pay for a substantial portion of healthcare costs through their contributions to employer-sponsored health insurance, and they are additionally impacted by indirect costs such as those due to disability and workplace absenteeism. Although opioid abuse has received widespread public attention in recent years, little is known about the economic costs of opioid abuse from an employer perspective.

The limited research that exists on the economic costs of opioid abuse suggests that these costs can be substantial for employers [8-10]. For example, Birnbaum et al. [8] estimated that, on a societal level, opioid abuse imposes an annual economic burden of US $\$ 55.7$ billion, with nearly half (US\$25.6 billion) of that total being due to workplace costs. In addition, using 2003-2007 data on commercially insured patients, White et al. [9] estimated that the average per-patient annual healthcare costs of patients diagnosed with abuse exceeded those of a comparison population by over US $\$ 20,000$. Those studies, however, used relatively few adjustments to control for a variety of potential differences between abusers and comparison patients. For example, it has been found that mental health conditions are both highly prevalent and associated with increased medical costs among abusers, and it is unclear to what extent the cost differentials found in those prior analyses were driven by abuse versus other underlying conditions that are disproportionately prevalent among abusers [11, 12]. In addition, the data used in those analyses pre-dated widespread private and public efforts to increase awareness of and reduce prescription opioid abuse and, as a result, they likely reflected neither the current prevalence of diagnosed abuse, which has increased nearly threefold over the 5-year period from 2006 to 2011 (Fig. 1), nor current abuse-related costs.
The objective of this study was to improve upon previous research by providing a more refined, current estimate of the excess healthcare and work-loss costs of prescription opioid abuse among commercially insured patients from 2006-2012. Excess costs of abuse were assessed using a study design that accounted for potential differences in age, sex, comorbidities and healthcare resource use between abusers and comparison patients. Annual incremental abuse-related costs, including healthcare and work-loss costs due to disability and medically related absenteeism, were calculated from the perspective of a self-insured employer.

\section{Methods}

\subsection{Data}

This study used de-identified administrative claims data from OptumHealth Reporting and Insights, a database containing information on approximately 16 million commercially insured beneficiaries (employees, spouses, children and retirees) from 60 large self-insured companies with locations across the USA, and representing a variety of industries and job types. The data included medical claims (dates of service, diagnoses received, procedures performed, places of service and payment amounts), pharmacy claims (fill dates, national drug codes [NDCs] and payment amounts), and eligibility information (patient demographics, enrolment history and wage information) for all beneficiaries for the period January 1999-March 2012. Data reporting work loss due to short- and long-term disability were available for employees from 33 of the 60 companies.

\subsection{Sample Selection}

Two mutually exclusive cohorts of commercially insured beneficiaries-including primary beneficiaries (i.e. employees) and dependents-were selected. Patients with abuse were identified as those with at least one of the following International Classification of Diseases, Ninth Revision, Clinical Modification (ICD-9-CM) codes for opioid abuse or dependence during the period January 2006-March 2012: 304.0x, 304.7x, 305.5x, 965.00, 965.02 and 965.09 [7]. As mentioned above, our use of both opioid abuse and dependence diagnosis codes is consistent with the NSDUH, which combines prescription pain reliever abuse and dependence [5]. This is also consistent with the Diagnostic and Statistical Manual of Mental Disorders, 5th edition (DSM-V), which combines categories of substance abuse and substance dependence into a single disorder [13]. In addition, clinical input suggests 
Fig. 1 Estimated prevalence of opioid abuse in the OptumHealth commercial claims database. The presence of diagnosed opioid use/ dependence was measured in the claims data as the proportion of covered lives (ages 12-64 years) with at least 1 month of eligibility in the calendar year that had at least one diagnosis for opioid use/ dependence in that year

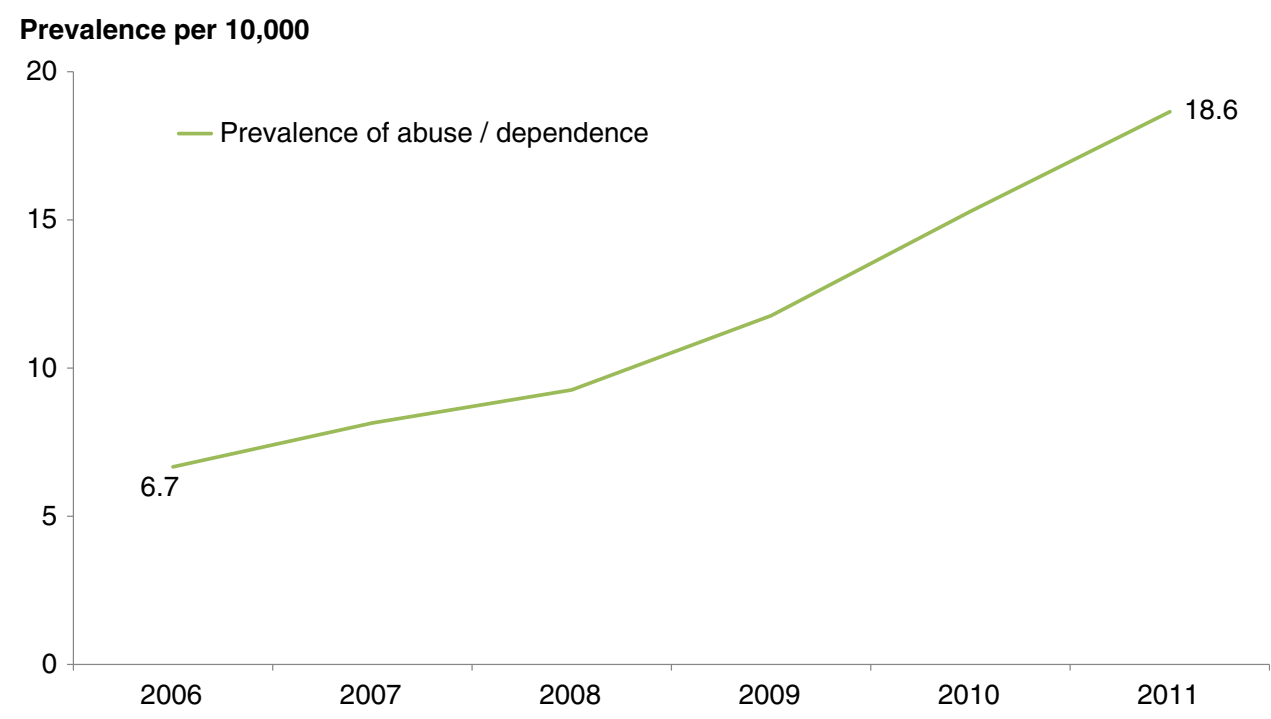

that opioid abuse and dependence diagnosis codes are often used interchangeably on insurance claims. A prior study on the excess costs of opioid abuse found that the magnitude of the excess costs was similar regardless of whether abuse-only or dependence-only codes were used [14]. Several other studies on opioid abuse have also used both opioid abuse and dependence diagnosis codes [7-9, 15]. Therefore, our cohort of 'abusers' contained patients diagnosed with abuse and/or dependence.

Because the objective of the analysis was to assess recent costs of abuse, patients with an abuse diagnosis prior to 2006 were excluded. The cohort of potential comparison patients was defined as all patients who did not have any of the aforementioned opioid abuse diagnoses at any time in their claims history (i.e. January 1999-March 2012). Because this resulted in a large comparator population, a $10 \%$ random sample was used for estimation purposes. For abusers, the index date was defined as the date of the first abuse diagnosis. For the comparison patients, the index date was assigned as the date of a random medical claim.

Each individual was observed over a 12-month followup period, which was centred on the index date and included the 6-month period prior to the index date, as abuse may result in medical costs prior to a formal diagnosis of abuse. This follow-up period was preceded by a 6-month baseline period, which was used for propensity score matching. Patients were required to be aged 12-64 years and continuously eligible with non-Health Maintenance Organization (non-HMO) coverage throughout the 18-month study period to ensure that all relevant drug and medical claims were captured for the final sample of patients. Patients aged 65 years and older were excluded from our study, as their Medicare eligibility may have limited our ability to observe all relevant drug and medical claims. These selection criteria resulted in a final analytic sample of 9,291 abusers and 395,901 comparison patients (Fig. 2).

\subsection{Prevalence of Diagnosed Abuse}

The prevalence rate of diagnosed abuse was calculated for each year from 2006 to 2011. The prevalence rate of diagnosed abuse in a given year was calculated as the proportion of covered lives (aged 12-64 years) with at least 1 month of eligibility in the calendar year that had at least one diagnosis for opioid abuse in that year.

\subsection{Propensity Score Matching}

Propensity score matching was used to account for observable differences at baseline between abusers and comparison patients. Propensity scores were estimated using logistic regression for all abusers and potential comparison patients on the basis of sex, age, US Census Division, selected baseline comorbidities that are likely to affect healthcare costs (e.g. cancer, congestive heart failure, mental health disorders) and baseline healthcare resource utilization (e.g. days hospitalized, days with an emergency department [ED] visit, prescription drug use). In order to ensure that patients were matched to those with availability of comparable treatment options and to facilitate the healthcare and work-loss cost comparisons, abusers were matched one-to-one with comparison patients on the basis of the year of the index date, baseline healthcare costs $( \pm 10 \%)$, availability of work-loss data and propensity score (within a quarter of a standard deviation [SD]), using greedy matching [16]. Full details of propensity score matching (including matching variables) are provided in the Technical Appendix. 
Fig. 2 Sample selection. $H M O$ Health Maintenance Organization, $Q 1$ quarter 1

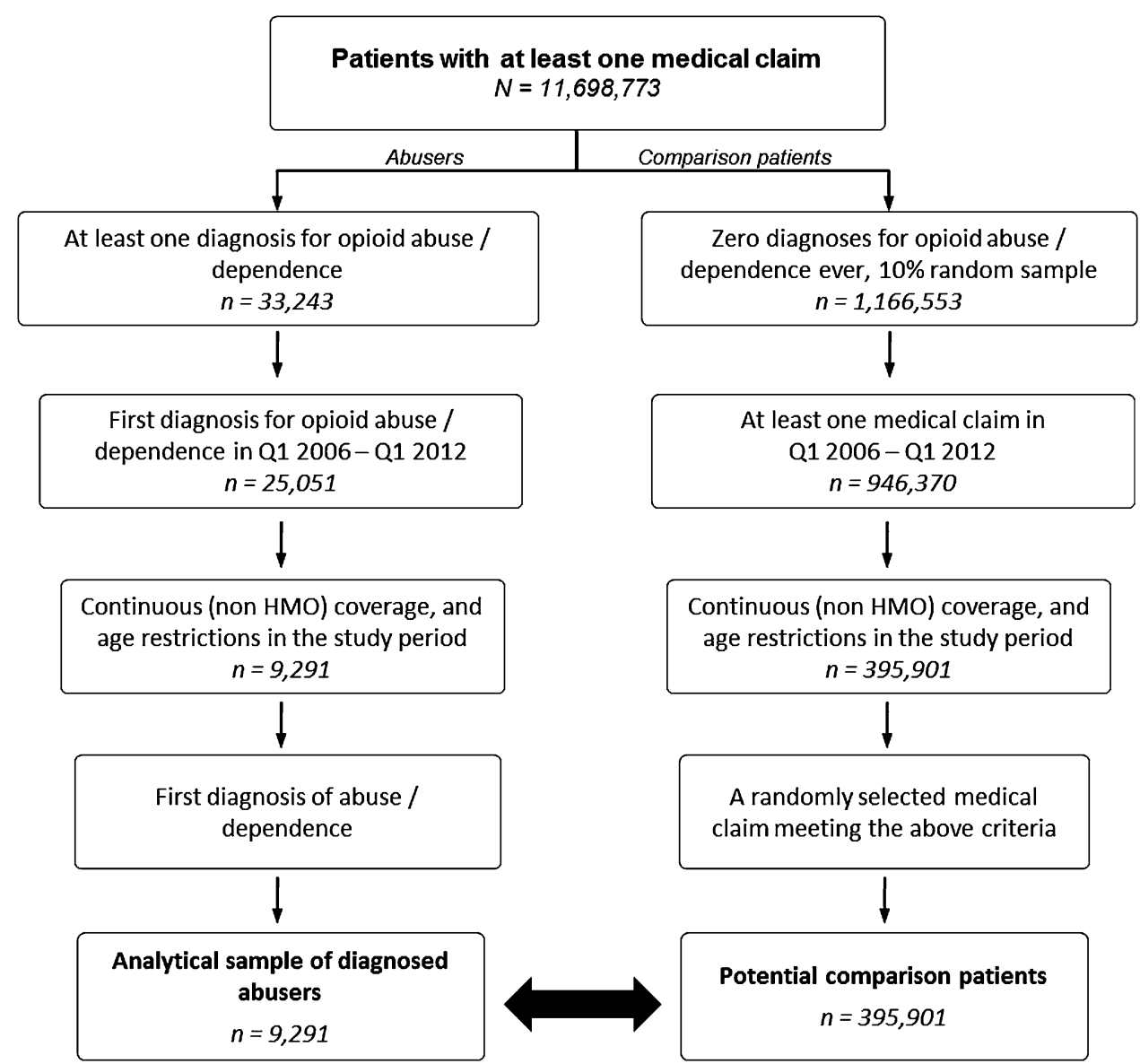

\subsection{Outcomes}

Total and incremental all-cause healthcare resource use and costs (as measured by payments made by third-party payers) in the 12-month follow-up period were compared for both abusers and comparison patients. Resource use and costs were categorized by the place of service (i.e. an inpatient facility, ED, outpatient/physician's office, rehabilitation facility, other [e.g. skilled nursing facility]) in order to understand the extent to which different types of healthcare resource utilization contribute to the cost differential between abusers and comparison patients. In addition, incremental indirect work-loss costs due to disability and medically related absenteeism were estimated for the subset of commercially insured patients for whom disability and wage information was available. For those patients, information regarding days and costs of missed work due to medical visits and/or disability were obtained directly from the database. Following Birnbaum et al. [8], medically related absenteeism was estimated by multiplying the number of days with medical resource use by the employee's wage: each ED visit accounted for a full day of missed work, the number of days hospitalized accounted for the equivalent number of full days of missed work and all other visits accounted for half a day of missed work each. This methodology for estimating medically related absenteeism has been used in other published studies estimating indirect work-loss costs [17, 18]. All costs were inflated to 2012 US dollars, using the Consumer Price Index [19].

Costs were also expressed in per-member per-month (PMPM) costs (which are sometimes referred to as 'perperson per-month' costs). The excess PMPM healthcare costs were calculated as the product of the prevalence of diagnosed opioid abuse and the per-patient excess healthcare costs. Similar calculations were conducted for the excess PMPM work-loss costs associated with diagnosed opioid abuse among individuals who were primary beneficiaries (as the work-loss costs of family members are not borne by a primary beneficiary's employer).

\subsection{Statistical Analyses}

For categorical variables, statistical significance was assessed using $\chi^{2}$ tests (pre-match) and McNemar tests (post-match). For continuous variables, statistical significance was assessed using Wilcoxon rank-sum tests (prematch) and Wilcoxon signed-rank tests (post-match). In 
addition, differences in mean costs post-matching were also evaluated using matched $t$ tests. Confidence intervals (CIs) were calculated using the means and standard errors of the reported statistics.

\section{Results}

\subsection{Prevalence of Diagnosed Abuse}

In 2011, the most recent year for which a full year of claims data were available, the prevalence of diagnosed abuse was 18.6 per 10,000 (95 \% CI 18.2-19.1 per 10,000) [Fig. 1]. There was a steady increase in the prevalence of diagnosed opioid abuse from 2006 (6.7 per 10,000, $95 \%$ CI 6.4-6.9 per 10,000) to 2011.

\subsection{Pre-match Baseline Characteristics}

Table 1 compares baseline characteristics between the abusers and comparison patients before and after matching. Before matching, abusers were statistically different $(p<0.001)$ from comparison patients on all demographic measures, with abusers being more likely to be male (54.5 versus $47.4 \%$; $p<0.001)$ and having different age and geographic distributions. There were also significant differences in the pre-match comorbidity profile and healthcare resource utilization for abusers and comparison patients. Specifically, the average Charlson Comorbidity Index (a score used to assess the general comorbidity burden) among abusers was over $80 \%$ higher than that of comparison patients ( 0.33 [SD 0.9] versus 0.18 [SD 0.6]; $p<0.001$ ), with abusers having at least twice the prevalence rate of comparison patients on 11 of the 17 conditions included in the index [20]. In addition, abusers were substantially more likely to have been diagnosed with abuse of other non-opioid substances (9.6 versus $0.9 \% ; p<0.001$ ), psychotic disorders $(16.2$ versus $2.7 \% ; p<0.001)$ or other mental health disorders $(27.7$ versus $7.2 \% ; p<0.001)$ in the baseline period.

As a result, abusers used significantly more healthcare resources, on average, in the baseline period, including days in an inpatient facility (1.0 day [SD 5.1] versus 0.1 days [SD 1.7]; $p<0.001)$ and $\mathrm{ED}$ (1.0 day [SD 3.5] versus 0.2 days [SD 1.1]; $p<0.001$ ). These differences resulted in average baseline healthcare costs among abusers being nearly four times those of comparison patients (US\$8,418 [SD US\$25,390] versus US\$2,129 [SD US\$9,503]; $p<0.001$ ).

\subsection{Post-match Follow-Up Period Resource Use and Costs}

Of the 9,291 abusers who met the initial selection criteria, the matching process resulted in identification of 7,658 matched pairs of abusers and comparison patients that were well balanced with respect to the baseline characteristics discussed above.

Despite having well-balanced baseline characteristics post-matching, abusers had significantly higher healthcare resource use and costs during the 12-month follow-up period (Table 2). Specifically, abusers spent an average of 6.2 more days in a drug rehabilitation facility and had, on average, 3.6 more days of hospitalization $(+491 \%), 1.7$ more ED visits $(+310 \%)$ and 5.3 more outpatient/physician office visits $(+137 \%)$ annually, compared with matched comparison patients. The average annual per-patient healthcare costs of abusers were US $\$ 20,343$ (SD US $\$ 36,498$ ), compared with US\$9,716 (SD US\$26,676) for matched comparison patients, yielding excess costs of US\$10,627 (95\% CI US $\$ 9,665-$ US $\$ 11,590)$. The main driver of the excess cost differential was inpatient costs, which accounted for approximately one third (US\$3,498) of the cost differential, followed by ED costs (US\$2,291) and rehabilitation facility costs (US\$1,772).

Among the subset of patients for whom work-loss data were available, abusers also missed significantly more work because of disability or medically related absenteeism during the follow-up period than matched comparison patients (Table 3), with an average of 6.3 more days on disability leave (15.5 [SD 51.7] versus 9.2 [SD 36.3]; $p=0.002)$ and 7.0 more days lost because of medically related absenteeism (13.4 [SD 19.3] versus 6.4 [SD 7.2]; $p<0.001$ ), for a total of 13.3 more days of missed work annually, on average. The average annual per-patient indirect work-loss costs, which included both disability and medically related absenteeism costs, were US\$3,773 (SD US\$6,648) for abusers and US $\$ 2,528$ (SD US $\$ 4,612$ ) for matched comparison patients, yielding excess indirect work-loss costs of US $\$ 1,244$ (95\% CI US\$790-US\$1,699).

Applying the 2011 prevalence of diagnosed opioid abuse $(18.6$ per 10,000) [Fig. 1] to the excess healthcare and work-loss costs discussed above, we estimated the costs of diagnosed opioid abuse from an employer perspective to be US\$1.71 PMPM, with US\$1.65 of that total comprising healthcare costs alone. In combining the healthcare and work-loss costs, it was necessary to account for the fact that not all covered beneficiaries were actively employed by the employer throughout the study period. The average portion of the year during which beneficiaries were employed (31\%) was used to scale down work-loss costs.

\section{Discussion}

Employers may not be aware of the substantial burden that opioid abuse imposes on them. The analyses described 
Table 1 Demographics, comorbidities and medical resource use in the baseline period ${ }^{\mathrm{a}}$

\begin{tabular}{|c|c|c|c|c|c|c|c|c|c|c|}
\hline & \multicolumn{5}{|c|}{ Unmatched sample } & \multicolumn{5}{|c|}{ Matched sample ${ }^{\mathrm{b}}$} \\
\hline & \multicolumn{2}{|l|}{ Abusers } & \multicolumn{2}{|c|}{$\begin{array}{l}\text { Comparison } \\
\text { patients }\end{array}$} & \multirow[t]{2}{*}{$p$ value $^{c}$} & \multicolumn{2}{|l|}{ Abusers } & \multicolumn{2}{|c|}{$\begin{array}{l}\text { Comparison } \\
\text { patients }\end{array}$} & \multirow[t]{2}{*}{$p$ value $^{\mathrm{d}}$} \\
\hline & $\%$ & $N$ & $\%$ & $N$ & & $\%$ & $N$ & $\%$ & $N$ & \\
\hline All patients & $100 \%$ & 9,291 & $100 \%$ & 395,901 & - & $100 \%$ & 7,658 & $100 \%$ & 7,658 & - \\
\hline $\begin{array}{l}\text { Age in years at index date [mean] } \\
\text { (SD) }\end{array}$ & {$[37.4]$} & (14.6) & {$[40.0]$} & $(15.5)$ & $<0.001$ & {$[36.5]$} & $(14.6)$ & {$[37.0]$} & $(16.3)$ & 0.015 \\
\hline Male sex & $54.5 \%$ & 5,063 & $47.4 \%$ & 187,705 & $<0.001$ & $56.4 \%$ & 4,321 & $54.7 \%$ & 4,186 & 0.013 \\
\hline \multicolumn{11}{|l|}{ US Census Division } \\
\hline East North Central & $19.3 \%$ & 1,795 & $17.4 \%$ & 68,703 & $<0.001$ & $18.6 \%$ & 1,425 & $20.5 \%$ & 1,573 & 0.001 \\
\hline Middle Atlantic & $18.4 \%$ & 1,706 & $14.5 \%$ & 57,456 & $<0.001$ & $18.7 \%$ & 1,435 & $19.2 \%$ & 1,473 & 0.398 \\
\hline South Atlantic & $17.6 \%$ & 1,639 & $18.9 \%$ & 74,902 & 0.002 & $18.3 \%$ & 1,398 & $16.7 \%$ & 1,279 & 0.007 \\
\hline West South Central & $10.4 \%$ & 966 & $13.3 \%$ & 52,497 & $<0.001$ & $10.3 \%$ & 792 & $10.0 \%$ & 767 & 0.482 \\
\hline Mountain & $9.5 \%$ & 887 & $7.3 \%$ & 28,950 & $<0.001$ & $9.3 \%$ & 709 & $9.5 \%$ & 730 & 0.528 \\
\hline Pacific & $8.2 \%$ & 763 & $9.2 \%$ & 36,386 & 0.001 & $8.0 \%$ & 612 & $7.6 \%$ & 579 & 0.291 \\
\hline East South Central & $7.4 \%$ & 685 & $5.5 \%$ & 21,793 & $<0.001$ & $7.5 \%$ & 575 & $7.0 \%$ & 536 & 0.192 \\
\hline West North Central & $4.8 \%$ & 450 & $9.0 \%$ & 35,608 & $<0.001$ & $4.8 \%$ & 368 & $5.0 \%$ & 380 & 0.632 \\
\hline New England & $4.2 \%$ & 390 & $4.5 \%$ & 17,973 & 0.117 & $4.4 \%$ & 334 & $4.3 \%$ & 330 & 0.867 \\
\hline Unknown & $0.1 \%$ & 10 & $0.4 \%$ & 1,633 & $<0.001$ & $0.1 \%$ & 10 & $0.1 \%$ & 11 & 0.819 \\
\hline \multicolumn{11}{|l|}{ Comorbidity profile } \\
\hline $\begin{array}{l}\text { Charlson Comorbidity Index } \\
\text { [mean] (SD) }\end{array}$ & {$[0.33]$} & $(0.9)$ & {$[0.18]$} & $(0.6)$ & $<0.001$ & {$[0.23]$} & $(0.7)$ & {$[0.25]$} & $(0.7)$ & 0.034 \\
\hline Chronic pulmonary disease & $7.1 \%$ & 657 & $3.1 \%$ & 12,328 & $<0.001$ & $5.3 \%$ & 406 & $5.4 \%$ & 412 & 0.827 \\
\hline Mild to moderate diabetes & $4.4 \%$ & 411 & $4.0 \%$ & 15,747 & 0.030 & $3.7 \%$ & 280 & $4.4 \%$ & 335 & 0.022 \\
\hline Rheumatological disease & $2.0 \%$ & 187 & $0.8 \%$ & 3,097 & $<0.001$ & $1.3 \%$ & 99 & $1.4 \%$ & 106 & 0.614 \\
\hline Congestive heart failure & $1.8 \%$ & 167 & $0.6 \%$ & 2,555 & $<0.001$ & $1.0 \%$ & 76 & $1.2 \%$ & 93 & 0.180 \\
\hline $\begin{array}{l}\text { Any malignancy, including } \\
\text { leukaemia and lymphoma }\end{array}$ & $1.7 \%$ & 156 & $1.5 \%$ & 6,091 & 0.277 & $1.3 \%$ & 98 & $1.5 \%$ & 115 & 0.237 \\
\hline Cerebrovascular disease & $1.7 \%$ & 155 & $0.6 \%$ & 2,513 & $<0.001$ & $1.1 \%$ & 82 & $1.3 \%$ & 97 & 0.251 \\
\hline $\begin{array}{l}\text { Diabetes with chronic } \\
\text { complications }\end{array}$ & $1.4 \%$ & 127 & $0.8 \%$ & 3,138 & $<0.001$ & $1.0 \%$ & 76 & $1.0 \%$ & 78 & 0.869 \\
\hline Peripheral vascular disease & $1.3 \%$ & 122 & $0.5 \%$ & 1,914 & $<0.001$ & $0.9 \%$ & 68 & $1.0 \%$ & 73 & 0.674 \\
\hline Mild liver disease & $1.0 \%$ & 91 & $0.3 \%$ & 1,059 & $<0.001$ & $0.7 \%$ & 52 & $0.7 \%$ & 50 & 0.843 \\
\hline Renal disease & $0.9 \%$ & 82 & $0.4 \%$ & 1,588 & $<0.001$ & $0.5 \%$ & 41 & $0.6 \%$ & 43 & 0.827 \\
\hline Peptic ulcer disease & $0.6 \%$ & 52 & $0.1 \%$ & 542 & $<0.001$ & $0.3 \%$ & 25 & $0.3 \%$ & 26 & 0.889 \\
\hline Myocardial infarction & $0.4 \%$ & 38 & $0.2 \%$ & 660 & $<0.001$ & $0.2 \%$ & 15 & $0.2 \%$ & 17 & 0.724 \\
\hline Hemiplegia or paraplegia & $0.4 \%$ & 36 & $0.1 \%$ & 319 & $<0.001$ & $0.2 \%$ & 17 & $0.2 \%$ & 15 & 0.724 \\
\hline Metastatic solid tumour & $0.3 \%$ & 29 & $0.2 \%$ & 706 & 0.003 & $0.1 \%$ & 11 & $0.2 \%$ & 16 & 0.336 \\
\hline HIV/AIDS & $0.3 \%$ & 27 & $0.2 \%$ & 622 & 0.001 & $0.2 \%$ & 17 & $0.2 \%$ & 14 & 0.590 \\
\hline Moderate or severe liver disease & $0.1 \%$ & 13 & $0.0 \%$ & 91 & $<0.001$ & $0.1 \%$ & 8 & $0.1 \%$ & 5 & 0.405 \\
\hline Dementia & $0.0 \%$ & 3 & $0.0 \%$ & 117 & 0.757 & $0.0 \%$ & 3 & $0.1 \%$ & 5 & 0.480 \\
\hline \multicolumn{11}{|l|}{ Other selected comorbidities } \\
\hline $\begin{array}{l}\text { Non-opioid substance abuse } \\
\text { diagnosis }\end{array}$ & $9.6 \%$ & 893 & $0.9 \%$ & 3,509 & $<0.001$ & $5.5 \%$ & 422 & $5.1 \%$ & 392 & 0.222 \\
\hline Psychotic disorder & $16.2 \%$ & 1,501 & $2.7 \%$ & 10,645 & $<0.001$ & $11.0 \%$ & 845 & $10.8 \%$ & 824 & 0.552 \\
\hline Other mental disorder & $27.7 \%$ & 2,572 & $7.2 \%$ & 28,645 & $<0.001$ & $21.8 \%$ & 1,672 & $23.4 \%$ & 1,790 & 0.008 \\
\hline \multicolumn{11}{|l|}{ Healthcare resource use [mean] (SD) } \\
\hline Number of inpatient days & {$[1.0]$} & $(5.1)$ & {$[0.1]$} & $(1.7)$ & $<0.001$ & {$[0.3]$} & $(2.3)$ & {$[0.3]$} & $(2.2)$ & 0.716 \\
\hline Number of ED days & [1.0] & $(3.5)$ & {$[0.2]$} & $(1.1)$ & $<0.001$ & {$[0.5]$} & $(1.4)$ & {$[0.4]$} & $(1.2)$ & $<0.001$ \\
\hline Number of outpatient visits & [8.3] & (9.7) & [3.7] & (5.9) & $<0.001$ & [6.1] & (7.4) & [6.6] & $(8.2)$ & $<0.001$ \\
\hline
\end{tabular}


Table 1 continued

\begin{tabular}{|c|c|c|c|c|c|c|c|c|c|c|}
\hline & \multicolumn{5}{|c|}{ Unmatched sample } & \multicolumn{5}{|c|}{ Matched sample ${ }^{b}$} \\
\hline & \multicolumn{2}{|l|}{ Abusers } & \multicolumn{2}{|c|}{$\begin{array}{l}\text { Comparison } \\
\text { patients }\end{array}$} & \multirow[t]{2}{*}{$p$ value $^{c}$} & \multicolumn{2}{|l|}{ Abusers } & \multicolumn{2}{|c|}{$\begin{array}{l}\text { Comparison } \\
\text { patients }\end{array}$} & \multirow[t]{2}{*}{$\overline{p \text { value }^{\mathrm{d}}}$} \\
\hline & $\%$ & $N$ & $\%$ & $N$ & & $\%$ & $N$ & $\%$ & $N$ & \\
\hline $\begin{array}{l}\text { Number of rehabilitation } \\
\text { facility days }\end{array}$ & {$[0.3]$} & $(4.1)$ & {$[0.0]$} & $(1.1)$ & $<0.001$ & {$[0.1]$} & $(2.2)$ & {$[0.1]$} & (1.9) & 0.303 \\
\hline Number of other visits & {$[1.2]$} & (3.7) & {$[0.5]$} & $(1.8)$ & $<0.001$ & {$[0.8]$} & $(2.4)$ & {$[0.8]$} & $(2.1)$ & 0.052 \\
\hline Number of prescriptions filled & {$[17.4]$} & $(19.9)$ & {$[5.4]$} & $(8.5)$ & $<0.001$ & [12.1] & $(13.6)$ & [11.4] & $(14.1)$ & $<0.001$ \\
\hline Number of unique NDCs filled ${ }^{\mathrm{e}}$ & [7.9] & $(8.1)$ & [2.9] & (3.9) & $<0.001$ & {$[5.7]$} & $(5.7)$ & {$[5.5]$} & (5.9) & $<0.001$ \\
\hline Total healthcare costs ${ }^{\mathrm{f}}$ & {$[\$ 8,418]$} & $(\$ 25,390)$ & {$[\$ 2,129]$} & $(\$ 9,503)$ & $<0.001$ & {$[\$ 3,485]$} & $(\$ 6,151)$ & {$[\$ 3,485]$} & $(\$ 6,151)$ & 0.510 \\
\hline
\end{tabular}

AIDS acquired immune deficiency syndrome, ED emergency department, $H I V$ human immunodeficiency virus, NDC National Drug Code, $S D$ standard deviation

a The baseline period was defined as the period between 12 months and 6 months prior to the index date

b Abusers were matched with potential comparison patients on the basis of the propensity score (within a quarter of a standard deviation), year of the index date, availability of work-loss data (0/1) and total healthcare costs (within $10 \%$ ) during the baseline period

${ }^{c} p$ values were calculated using $\chi^{2}$ tests for binary variables and Wilcoxon rank-sum tests for continuous variables

${ }^{\mathrm{d}} p$ values were calculated using McNemar tests for matched pairs for binary variables and Wilcoxon signed-rank tests for continuous variables

e The numbers of unique NDCs filled were identified using 9-digit NDCs

${ }^{\mathrm{f}}$ Healthcare costs were inflated to 2012 dollars, using the medical care component of the Consumer Price Index. The costs per patient reflect the amount paid by the insurer, excluding out-of-pocket payments by patients (e.g. copay, deductibles). The numbers may not sum to the total, because of rounding

above examined the excess costs of opioid abuse and highlight the substantial burden imposed on employers, including both direct healthcare costs and indirect workloss costs. We found that a diagnosed abuser had, on average, excess annual healthcare costs of US\$10,627 and US\$1,244 in excess annual work-loss costs. Although previous papers have estimated the costs of opioid abuse, to our knowledge this is the first study to assess excess costs from the employer perspective using a refined set of controls to account for significantly higher underlying rates of comorbidities and baseline healthcare resource use. Efforts to educate employees about warning signs of opioid abuse may help employers reduce the burden of opioid abuse by preventing opioid abuse or helping abusers to obtain treatment sooner.

The excess PMPM healthcare costs of diagnosed opioid abuse of US\$1.65 represent substantial costs to employers that are comparable to the costs of several costly mental health conditions. For example, the excess PMPM healthcare costs of diagnosed opioid abuse are higher than the published PMPM healthcare costs of schizophrenia (US\$0.30) and anxiety disorder (US\$0.97), and are similar to the PMPM healthcare costs of depression (US\$1.73) and alcoholism (US\$2.00) [Table 4] [21].

While the excess costs of diagnosed opioid abuse observed in claims data indicate a substantial burden to employers, they nonetheless understate the overall costs of opioid abuse to employers because they fail to account for the large portion of opioid abusers who remain undiagnosed. A preliminary calculation suggests that the costs associated with undiagnosed abuse may indeed exceed those of diagnosed abuse. In order to obtain a preliminary estimate of the prevalence of undiagnosed abuse, we combined our claims data analyses of diagnosed abusers with published government data from the NSDUH on the overall rate of diagnosed and undiagnosed abuse in the USA in 2011 (the most recent year for which a complete year of claims data were available). In the absence of an estimate of undiagnosed abuse in the literature, the difference between the overall rate of abuse reported in the NSDUH $(80$ per 10,000) and the rate of diagnosed abuse calculated in our claims data analysis (18.6 per 10,000) provides a first approximation of the rate of undiagnosed abuse in the USA $(61.4$ per 10,000) $[6,22,23]$. This rate of undiagnosed abuse implies a ratio of 3.3 undiagnosed abusers per diagnosed abuser in the USA.

Combining the preliminary prevalence estimates with the estimated costs of undiagnosed abuse, which prior literature has estimated to be $80 \%$ of the costs of diagnosed abuse [15], suggests that undiagnosed opioid abuse may contribute US\$4.36 in additional excess PMPM healthcare costs and US\$0.16 in additional excess PMPM work-loss costs. Excess PMPM healthcare costs of this magnitude represent a substantial burden to employers and would be comparable to published estimates of the PMPM healthcare costs associated with colorectal cancer (US\$4.38), 
Table 2 Healthcare resource use and costs among matched abusers $(N=7,658)$ and comparison patients $(N=7,658)$ in the follow-up period ${ }^{\text {a,b }}$

\begin{tabular}{|c|c|c|c|c|c|c|c|}
\hline & \multicolumn{2}{|l|}{ Abusers } & \multicolumn{2}{|c|}{ Comparison patients } & \multirow[t]{2}{*}{ Difference } & \multirow[t]{2}{*}{ Ratio } & \multirow[t]{2}{*}{$p$ value } \\
\hline & Mean & SD & Mean & SD & & & \\
\hline \multicolumn{8}{|l|}{ Healthcare resource use } \\
\hline \multicolumn{8}{|l|}{ Medical resource use } \\
\hline Number of inpatient days & 4.5 & 11.0 & 0.9 & 5.6 & 3.6 & 4.9 & $<0.001$ \\
\hline Number of ED days & 2.5 & 4.7 & 0.8 & 2.4 & 1.7 & 3.1 & $<0.001$ \\
\hline Number of outpatient visits & 19.7 & 17.3 & 14.4 & 15.6 & 5.3 & 1.4 & $<0.001$ \\
\hline Number of rehabilitation facility days & 6.5 & 19.2 & 0.2 & 2.9 & 6.2 & 31.2 & $<0.001$ \\
\hline Number of other visits & 3.1 & 6.4 & 1.9 & 5.5 & 1.1 & 1.6 & $<0.001$ \\
\hline \multicolumn{8}{|l|}{ Prescription drug use } \\
\hline Number of prescriptions filled & 31.6 & 30.6 & 22.4 & 27.7 & 9.2 & 1.4 & $<0.001$ \\
\hline Number of unique NDCs filled ${ }^{\mathrm{d}}$ & 13.3 & 12.8 & 8.5 & 8.8 & 4.8 & 1.6 & $<0.001$ \\
\hline \multicolumn{8}{|l|}{ Healthcare costs ${ }^{\mathrm{e}}$} \\
\hline Total healthcare costs & $\$ 20,343$ & $\$ 36,498$ & $\$ 9,716$ & $\$ 26,676$ & $\$ 10,627$ & 2.1 & $<0.001$ \\
\hline Medical costs & $\$ 17,518$ & $\$ 35,488$ & $\$ 7,671$ & $\$ 25,639$ & $\$ 9,847$ & 2.3 & $<0.001$ \\
\hline Inpatient costs & $\$ 5,843$ & $\$ 25,952$ & $\$ 2,345$ & $\$ 18,932$ & $\$ 3,498$ & 2.5 & $<0.001$ \\
\hline ED costs & $\$ 3,163$ & $\$ 9,880$ & $\$ 871$ & $\$ 5,232$ & $\$ 2,291$ & 3.6 & $<0.001$ \\
\hline Outpatient costs & $\$ 5,465$ & $\$ 10,532$ & $\$ 3,997$ & $\$ 11,483$ & $\$ 1,467$ & 1.4 & $<0.001$ \\
\hline Rehabilitation facility costs & $\$ 1,821$ & $\$ 5,904$ & $\$ 49$ & $\$ 825$ & $\$ 1,772$ & 36.9 & $<0.001$ \\
\hline Other costs & $\$ 1,227$ & $\$ 4,681$ & $\$ 408$ & $\$ 4,541$ & $\$ 819$ & 3.0 & $<0.001$ \\
\hline Prescription drug costs & $\$ 2,826$ & $\$ 5,169$ & $\$ 2,045$ & $\$ 4,287$ & $\$ 781$ & 1.4 & $<0.001$ \\
\hline
\end{tabular}

$E D$ emergency department, $N D C$ National Drug Code, $S D$ standard deviation

a The 12-month follow-up period was defined as the 6 months prior to and the 6 months following the index date. The numbers may not sum to the total, because of rounding

b Abusers were matched with potential comparison patients on the basis of the propensity score (within a quarter of a standard deviation), year of the index date, availability of work loss data (0/1) and total healthcare costs (within $10 \%$ ) during the baseline period

c $p$ values were calculated using McNemar tests for matched pairs for binary variables and Wilcoxon signed-rank tests for continuous variables

${ }^{d}$ The numbers of unique NDCs filled were identified using 9-digit NDCs

${ }^{\text {e }}$ Healthcare costs were inflated to 2012 US dollars, using the medical care component of the Consumer Price Index. The costs per patient reflect the amount paid by the insurer, excluding out-of-pocket payments by patients (e.g. copay, deductibles)

chronic obstructive pulmonary disease (US\$5.35), obesity (US\$4.84-US\$7.78) and osteoarthritis (US\$6.64) [Table 4] [21, 24]. Given the magnitude of potential costs associated with undiagnosed abuse, further research should attempt to validate the preliminary estimate of the prevalence of undiagnosed abuse, as well as the assumption that the costs of undiagnosed abuse are $80 \%$ of the costs of diagnosed abuse.

This study had a number of limitations. First, the analysis relied on the accuracy of claims data to distinguish abusers from comparison patients, evaluate their comorbidity profiles at baseline and evaluate healthcare resource use and cost information during the follow-up period. Any miscoding in the underlying data could have affected our results, although we have no reason to believe that any inaccuracies in the data affected the abusers or comparison patients differently.

Second, and relatedly, because undiagnosed opioid abusers do not receive any of the ICD-9-CM diagnosis codes for abuse by definition, we expect that some of these patients were actually included in our cohort of comparison patients. While the extent to which this is the case is unknown, if undiagnosed abusers are more costly than a true comparison population comprising patients who are not abusers, this would imply that the estimated excess costs of diagnosed abuse understate the actual excess cost differential between abusers and a comparison population of patients without diagnosed or undiagnosed opioid abuse.

Third, the calculations of incremental healthcare and work-loss costs were based on those of patients with 18 months of continuous eligibility. This requirement was necessary in order to ensure complete visibility of medical and prescription drug claims during the study period. However, this criterion could have excluded more severe abusers, who may be less capable of maintaining constant employment and health insurance eligibility. This would imply that our estimates of the burden of opioid abuse are an underestimate. 
Table 3 Medically related absenteeism, disability and related costs among matched abusers $(N=1,162)$ and comparison patients $(N=1,162)$ in the follow-up period ${ }^{\mathrm{a}, \mathrm{b}}$

\begin{tabular}{|c|c|c|c|c|c|c|c|}
\hline & \multicolumn{2}{|l|}{ Abusers } & \multicolumn{2}{|c|}{ Comparison patients } & \multirow[t]{2}{*}{ Difference } & \multirow[t]{2}{*}{ Ratio } & \multirow[t]{2}{*}{$p$ value } \\
\hline & Mean & SD & Mean & SD & & & \\
\hline \multicolumn{8}{|l|}{ Work loss } \\
\hline Patients with any work-loss day [\%] $(n)$ & {$[99.9 \%]$} & $(1,161)$ & [99.1\%] & $(1,152)$ & {$[0.8 \%]$} & 1.0 & 0.007 \\
\hline Patients with disability & {$[16.6 \%]$} & $(193)$ & {$[12.0 \%]$} & $(139)$ & {$[4.6 \%]$} & 1.4 & 0.001 \\
\hline Patients with medically related absenteeism ${ }^{\mathrm{d}}$ & {$[96.5 \%]$} & $(1,121)$ & [97.8\%] & $(1,137)$ & {$[-1.4 \%]$} & 1.0 & 0.046 \\
\hline Number of work-loss days & 28.9 & 52.5 & 15.6 & 36.7 & 13.3 & 1.8 & $<0.001$ \\
\hline Number of disability days & 15.5 & 51.7 & 9.2 & 36.3 & 6.3 & 1.7 & 0.002 \\
\hline Number of medically related absenteeism days & 13.4 & 19.3 & 6.4 & 7.2 & 7.0 & 2.1 & $<0.001$ \\
\hline \multicolumn{8}{|l|}{ Work-loss costs ${ }^{\mathrm{e}, \mathrm{f}}$} \\
\hline Total work-loss costs & $\$ 3,773$ & $\$ 6,648$ & $\$ 2,528$ & $\$ 4,612$ & $\$ 1,244$ & 1.5 & $<0.001$ \\
\hline Disability costs & $\$ 1,378$ & $\$ 5,347$ & $\$ 873$ & $\$ 3,600$ & $\$ 505$ & 1.6 & 0.007 \\
\hline Medically related absenteeism costs & $\$ 2,395$ & $\$ 4,305$ & $\$ 1,655$ & $\$ 2,840$ & $\$ 739$ & 1.4 & $<0.001$ \\
\hline
\end{tabular}

$E D$ emergency department, $S D$ standard deviation

a The 12-month follow-up period was defined as the 6 months prior to and the 6 months following the index date. The numbers may not sum to the total, because of rounding

b Abusers were matched with potential comparison patients on the basis of the propensity score (within a quarter of a standard deviation), year of the index date, availability of work loss data (0/1) and total healthcare costs (within $10 \%$ ) during the baseline period

${ }^{c} p$ values were calculated using McNemar tests for matched pairs for binary variables and Wilcoxon signed-rank tests for continuous variables

${ }^{\mathrm{d}}$ Medically related absenteeism was calculated using medical claims occurring during the work week. Days with a hospitalization or ED visit were counted as a full day of absenteeism, and all other visits were counted as a half day of absenteeism, following Birnbaum et al. [8]

e Disability costs were obtained directly from the database. Medically related absenteeism costs were calculated by multiplying the number of days of missed work by the employee's daily wage

f Total work-loss costs were inflated to 2012 US dollars, using the all-items component of the Consumer Price Index

Table 4 Comparison of per-member per-month (PMPM) healthcare costs

\begin{tabular}{|c|c|c|}
\hline Condition & $\begin{array}{l}\text { PMPM } \\
\text { costs }^{\mathrm{a}}\end{array}$ & Source \\
\hline $\begin{array}{l}\text { Schizophrenia, } \\
\text { acute }\end{array}$ & $\$ 0.30$ & Goetzel et al. [21] \\
\hline Anxiety disorder & $\$ 0.97$ & Goetzel et al. [21] \\
\hline $\begin{array}{r}\text { Opioid abuse } \\
\text { (diagnosed) }\end{array}$ & $\$ 1.65$ & $\begin{array}{l}\text { Authors' calculation based on } \\
\text { OptumHealth Reporting and } \\
\text { Insights claims data analysis }\end{array}$ \\
\hline Depression & $\$ 1.73$ & Goetzel et al. [21] \\
\hline Alcoholism & $\$ 2.00$ & Goetzel et al. [21] \\
\hline Colorectal cancer & $\$ 4.38$ & Goetzel et al. [21] \\
\hline Obesity (in men) & $\$ 4.84$ & Long et al. [24] \\
\hline $\begin{array}{l}\text { Chronic obstructive } \\
\text { pulmonary disease }\end{array}$ & $\$ 5.35$ & Goetzel et al. [21] \\
\hline Obesity (in women) & $\$ 7.78$ & Long et al. [24] \\
\hline Osteoarthritis & $\$ 6.64$ & Goetzel et al. [21] \\
\hline Hypertension & $\$ 13.15$ & Goetzel et al. [21] \\
\hline
\end{tabular}

a All costs were inflated to 2012 US dollars, using the medical care services component of the Consumer Price Index (December figures)

Fourth, while propensity score matching led to matched cohorts having similar baseline characteristics, approximately $20 \%$ of abusers were excluded. These included some high-cost patients with higher rates of comorbidities and medical resource use from the analysis (Table 1), possibly suggesting that the estimate of excess costs was conservative.

Fifth, the analysis did not include certain cost categories that are relevant to employers, including sick time spent at home (i.e. the analysis only accounted for time lost because of healthcare visits), reduced on-thejob productivity or costs associated with potential turnover of some individuals (e.g. administrative costs of identifying and training new employees). Also, the workloss estimates were based on an algorithm that calculates days missed as a function of medical resource utilization, and we did not observe the extent to which certain employees may have compensated for medically related absenteeism (e.g. by working on weekends). As a result, the actual indirect work-loss costs of opioid abuse borne by employers may differ from those reported here. Finally, we assumed that the prevalence and costs of diagnosed abuse calculated in the OptumHealth claims data were representative of the prevalence and costs of diagnosed abuse of the broader population covered by self-insured employers. Future research should validate these findings in other commercial claims databases. 


\section{Conclusion}

This study has provided an up-to-date and refined estimate of the annual incremental costs of diagnosed opioid abuse, using a large administrative claims database, controlling for a broad array of underlying differences between opioid abusers and comparison patients. Many payers in the healthcare industry are unaware of the substantial burden of prescription opioid abuse [25]. However, our study has demonstrated that there are substantial costs of abuse to employers, who pay for a large portion of the healthcare costs of opioid abusers. Employers also incur costs of work loss and diminished workplace productivity due to opioid abuse. These results suggest that in addition to recent and widespread government and regulatory initiatives in this therapeutic area, it may be beneficial for employers, for whom the full burden of opioid abuse is potentially hidden, to take proactive steps to address the issue through educational programmes on the warning signs of opioid abuse and coverage of broad options for treatment.

Acknowledgments This study was funded by Purdue Pharma L.P. The authors Bradford Rice, Noam Kirson, Amie Shei, Alice Kate Cummings, Katharine Bodnar and Howard Birnbaum are employees of Analysis Group, Inc., a company that received funding from Purdue Pharma L.P. to conduct this study. Rami Ben-Joseph is an employee of Purdue Pharma L.P.

Author contributions Bradford Rice, Noam Kirson, Amie Shei, Howard Birnbaum and Rami Ben-Joseph were the primary developers of the study design. The data analysis was done primarily by Alice Kate Cummings and Katharine Bodnar. All authors reviewed and discussed the study results, and contributed to writing and editing the manuscript. Bradford Rice is the guarantor of this work and, as such, had full access to all of the data in the study and takes responsibility for the integrity of the data and the accuracy of the data analysis.

\section{Technical Appendix}

As discussed above, propensity score matching was used to account for observable differences at baseline between abusers and comparison patients. Specifically, a logistic regression model was used, with the dependent variable being an indicator of whether a patient was diagnosed with opioid abuse or dependence. Independent variables included the following patients and baseline characteristics:

- Age (years)

- Sex indicator

- Indicators for US Census Divisions

- Indicator variables for diagnoses of selected baseline comorbidities

- Myocardial infarction; congestive heart failure; peripheral vascular disease; cerebrovascular
Table 5 Propensity score logistic regression model results

\begin{tabular}{|c|c|c|c|}
\hline Parameter & Coefficient & SE & $p$ value \\
\hline Intercept & -5.23 & 0.47 & $<0.001$ \\
\hline \multicolumn{4}{|l|}{ Demographic characteristics } \\
\hline Age & -0.03 & 0.00 & $<0.001$ \\
\hline Male sex & -0.32 & 0.01 & $<0.001$ \\
\hline \multicolumn{4}{|l|}{ US Census Division } \\
\hline Unknown & -1.10 & 0.29 & $<0.001$ \\
\hline 1: New England & 0.11 & 0.06 & 0.064 \\
\hline 2: Middle Atlantic & 0.47 & 0.04 & $<0.001$ \\
\hline 3: East North Central & 0.21 & 0.04 & $<0.001$ \\
\hline 4: West North Central & -0.54 & 0.06 & $<0.001$ \\
\hline 5: South Atlantic & 0.10 & 0.04 & 0.013 \\
\hline 6: East South Central & 0.34 & 0.05 & $<0.001$ \\
\hline 7: West South Central & -0.13 & 0.05 & 0.004 \\
\hline 8: Mountain & 0.45 & 0.05 & $<0.001$ \\
\hline \multicolumn{4}{|l|}{ Comorbidities } \\
\hline Chronic pulmonary disease & 0.11 & 0.02 & $<0.001$ \\
\hline Mild to moderate diabetes & 0.40 & 0.03 & $<0.001$ \\
\hline Rheumatological disease & 0.04 & 0.05 & 0.344 \\
\hline Congestive heart failure & 0.17 & 0.05 & 0.001 \\
\hline $\begin{array}{l}\text { Any malignancy, including } \\
\text { leukaemia and lymphoma }\end{array}$ & 0.19 & 0.05 & $<0.001$ \\
\hline Cerebrovascular disease & 0.02 & 0.05 & 0.722 \\
\hline Diabetes with chronic complications & 0.55 & 0.06 & $<0.001$ \\
\hline Peripheral vascular disease & -0.03 & 0.06 & 0.591 \\
\hline Mild liver disease & -0.21 & 0.07 & 0.001 \\
\hline Renal disease & 0.31 & 0.07 & $<0.001$ \\
\hline Peptic ulcer disease & -0.10 & 0.09 & 0.298 \\
\hline Myocardial infarction & 0.24 & 0.11 & 0.022 \\
\hline Hemiplegia or paraplegia & -0.09 & 0.11 & 0.421 \\
\hline Metastatic solid tumour & 0.20 & 0.11 & 0.073 \\
\hline HIV/AIDS & 0.30 & 0.11 & 0.007 \\
\hline Moderate or severe liver disease & -0.02 & 0.20 & 0.900 \\
\hline Dementia & 0.92 & 0.31 & 0.003 \\
\hline $\begin{array}{l}\text { Non-opioid substance abuse } \\
\text { diagnosis }\end{array}$ & -0.84 & 0.02 & $<0.001$ \\
\hline Psychotic disorder & -0.27 & 0.02 & $<0.001$ \\
\hline Other mental disorder & -0.36 & 0.01 & $<0.001$ \\
\hline \multicolumn{4}{|l|}{ Baseline healthcare resource use } \\
\hline Inpatient days & 0.01 & 0.00 & 0.001 \\
\hline ED days & 0.05 & 0.00 & $<0.001$ \\
\hline Outpatient visits & 0.01 & 0.00 & $<0.001$ \\
\hline Rehabilitation facility days & 0.01 & 0.00 & 0.064 \\
\hline Other visits & -0.02 & 0.00 & $<0.001$ \\
\hline Number of prescriptions filled & 0.04 & 0.00 & $<0.001$ \\
\hline Number of unique NDCs filled & 0.07 & 0.00 & $<0.001$ \\
\hline
\end{tabular}

The sample size was 405,192

$A I D S$ acquired immune deficiency syndrome, ED emergency department, HIV human immunodeficiency virus, NDC National Drug Code, $S E$ standard error 
disease; dementia; chronic pulmonary disease; rheumatological disease; peptic ulcer disease; mild liver disease; mild to moderate diabetes; diabetes with chronic complications; hemiplegia or paraplegia; renal disease; any malignancy, including leukaemia and lymphoma; moderate or severe liver disease; metastatic solid tumour; HIV/AIDS; psychotic disorders; non-opioid substance abuse diagnoses; other mental disorders

\section{- Baseline healthcare resource utilization}

- Number of inpatient days; number of ED days; number of outpatient visits; number of rehabilitation facility days; number of other visits

- Number of unique NDCs filled; number of prescriptions filled

Appendix Table 5 summarizes the results of the logistic regression model used to estimate the propensity scores.

The model yielded a c-statistic of 0.81 . Following propensity score estimation, abusers were matched one-to-one with comparison patients on the basis of the year of the index date, baseline healthcare costs $( \pm 10 \%)$, availability of work-loss data and propensity score (within a quarter of a standard deviation), using greedy matching. The year of the index date was meant to account for possible differences over time in treatment practices. Baseline healthcare costs were used as a measure of severity to ensure that there were no underlying differences in the intensity of healthcare resource use at baseline. Inclusion of baseline healthcare costs in the propensity score model was examined and was found to result in a poorer match at baseline and greater study period excess costs of abuse. The availability of work-loss data was important to include in the direct match to allow for consistent examination of the subset of patients with work-loss data.

\section{References}

1. Institute of Medicine. Relieving pain in America: a blueprint for transforming prevention, care, education, and research. Washington, DC: National Academies Press, 2011.

2. Stewart WF, Ricci JA, Chee E, Morganstein D, Lipton R. Lost productive time and cost due to common pain conditions in the US workforce. JAMA. 2003;290:2443-54.

3. Volkow ND, McLellan TA. Curtailing diversion and abuse of opioid analgesics without jeopardizing pain treatment. JAMA. 2011;305:1346-7.

4. IMS Institute for Healthcare Informatics. The use of medicines in the United States: review of 2011. April 2012. http://www. imshealth.com/ims/Global/Content/Insights/IMS\%20Institute\% 20for\%20Healthcare\%20Informatics/IHII_Medicines_in_U.S_ Report_2011.pdf. Accessed 1 Sept 2013.

5. Substance Abuse and Mental Health Services Administration. Results from the 2006 National Survey on Drug Use and Health: national findings. Rockville: Substance Abuse and Mental Health Services Administration, 2007. http://www.samhsa.gov/data/ nsduh/2k6nsduh/2k6results.pdf. Accessed 1 Aug 2013.

6. Substance Abuse and Mental Health Services Administration. Results from the 2011 National Survey on Drug Use and Health: summary of national findings. Rockville: Substance Abuse and Mental Health Services Administration, 2012. http://www. samhsa.gov/data/NSDUH/2k11Results/NSDUHresults2011.htm. Accessed 1 Aug 2013.

7. Rice JB, White AG, Birnbaum HG, Schiller M, Brown DA, Roland CL. A model to identify patients at risk for prescription opioid abuse, dependence, and misuse. Pain Med. 2012;13: $1162-73$.

8. Birnbaum HG, White AG, Schiller M, Waldman T, Cleveland JM, Roland CL. Societal costs of prescription opioid abuse, dependence, and misuse in the United States. Pain Med. 2011;12:657-67.

9. White AG, Birnbaum HG, Schiller M, Waldman T, Cleveland JM, Roland CL. Economic impact of opioid abuse, dependence, and misuse. Am J Pharm Benefits. 2011;3:e59-70.

10. Strassels SA. Economic burden of prescription opioid misuse and abuse. J Manag Care Pharm. 2009;15(7):556-62.

11. McAdam-Marx C, Roland CL, Cleveland J, Oderda GM. Costs of opioid abuse and misuse determined from a Medicaid database. J Pain Palliat Care Pharmacother. 2010;24(1):5-18.

12. Becker WC, Sullivan LE, Tetrault JM, Desai RA, Fiellin DA. Non-medical use, abuse and dependence on prescription opioids among US adults: psychiatric, medical and substance use correlates. Drug Alcohol Depend. 2008;94:38-47.

13. Substance-Related and Addictive Disorders. American Psychiatric Association. http://www.dsm5.org/Documents/Substance\% 20Use\%20Disorder\%20Fact\%20Sheet.pdf. Accessed 21 Mar 2014.

14. White AG, Birnbaum HG, Mareva MN, et al. Direct costs of opioid abuse in an insured population in the United States. J Manag Care Pharm. 2005;11:469-79.

15. White AG, Birnbaum HG, Rothman DB, Katz N. Development of a budget-impact model to quantify potential cost savings from prescription opioids designed to deter abuse or ease of extraction. Appl Health Econ Policy. 2009;7:61-70.

16. Austin PC. An introduction to propensity score methods for reducing the effects of confounding in observational studies. Multivar Behav Res. 2011;46:399-424.

17. Rice JB, Desai U, Cummings AKG, Birnbaum HB, Skornicki M, Parsons N. Burden of diabetic foot ulcers for Medicare and private insurers. Diabetes Care. 2014;37:651-8.

18. Johnson SJ, Kaltenboek A, Diener M, Birnbaum HG, Grubb E, Castelli-Haley J, Siderowf AD. Costs of Parkinson's disease in a privately insured population. Pharmacoeconomics. 2013;31: 799-806.

19. US Bureau of Labor Statistics. Consumer Price Index. Washington DC, 2013. http://www.bls.gov/cpi/home.htm. Accessed 8 May 2014.

20. Romano PS, Roos LL, Jollis JG. Adapting a clinical comorbidity index for use with ICD-9-CM administrative data: differing perspectives. J Clin Epidemiol. 1993;46(10):1075-9.

21. Goetzel RZ, Hawkins K, Ozminkowski RJ, Wang S. The health and productivity cost burden of the "top 10" physical and mental health conditions affecting six large US employers in 1999. J Occup Environ Med. 2003;45(1):5-14.

22. Substance Abuse and Mental Health Services Administration. Results from the 2010 National Survey on Drug Use and Health: summary of national findings. Rockville: Substance Abuse and Mental Health Services Administration, 2011. http://www.samhsa. gov/data/NSDUH/2k10ResultsRev/NSDUHresultsRev2010.htm. Accessed 16 Jan 2014. 
23. Substance Abuse and Mental Health Services Administration. Results from the 2012 National Survey on Drug Use and Health: summary of national findings. Rockville: Substance Abuse and Mental Health Services Administration, 2013. http:// www.samhsa.gov/data/NSDUH/2012SummNatFindDetTables/ NationalFindings/NSDUHresults2012.htm. Accessed 16 Jan 2014.
24. Long DA, Reed R, Lehman G. The cost of lifestyle health risks: obesity. J Occup Environ Med. 2006;48:244-51.

25. Katz NP, Birnbaum H, Brennan MJ, Freedman JD, Gilmore GP, Jay D, Kenna GA, Madras BK, McElhaney L, Weiss RD, White AG. Prescription opioid abuse: challenges and opportunities for payers. Am J Manag Care. 2013;19(4):295-302. 